\title{
Using MOOCs for teaching analytical chemistry: experience at University of Tartu
}

\author{
Ivo Leito • Irja Helm • Lauri Jalukse
}

Published online: 11 January 2015

(C) Springer-Verlag Berlin Heidelberg 2015

\section{Introduction}

The term MOOC (massive open online course) was coined in 2008 by Dave Cormier and George Siemens [1]. Since then, MOOCs have attracted much attention and public opinion on MOOCs has changed remarkably during the last few years. At the beginning of this decade MOOCs were hailed as the future of higher education and the year 2012 was declared by the New York Times as "the year of the MOOC" [2]. It was even envisaged that higher education as we know it may collapse. Since then MOOCs have received quite some criticismbased on e.g. insufficient interaction between teachers and students, low course completion rates, etc. [3] - and it is now widely acknowledged that MOOCs were originally overhyped [4]. Nevertheless, MOOCs are offered by numerous universities, as well as by several major MOOC providers, such as Coursera [5] or edX [6]. The intense development of MOOCs goes on and rightly so, because MOOCs obviously enrich the higher education possibilities in the world.

MOOCs are intrinsically less suited for experimental sciences, compared to e.g. web design, history or business, because it is impossible to offer the experimental/laboratory training via the Web. Nevertheless, there are MOOCs available also in chemistry and, more specifically, in analytical chemistry. The course "Analytical chemistry/instrumental analysis" (Prof. Vicki Colvin, Rice University) is offered at Coursera, and Udemy offers the course "Analytical chemistry" (Oxford Royale Academy).

In this paper we present the experience of running a MOOC "Estimation of measurement uncertainty in chemical analysis" [7] at the University of Tartu (UT). We compare

I. Leito $(\bowtie) \cdot$ I. Helm $\cdot$ L. Jalukse

Institute of Chemistry, University of Tartu, Ravila 14a, 50411 Tartu, Estonia

e-mail: ivo.leito@ut.ee teaching in the "MOOC mode" to conventional university teaching as well as to short training courses for professionals.

Description of the course

The course consists of the course materials on the Web [7] and learning management system in the UT Moodle environment. It is an introductory course on estimation of measurement uncertainty (MU) (note, in this paper the terms "estimation of MU" and "evaluation of MU" are used as synonyms), specifically related to chemical analysis (analytical chemistry) with volume of 1 ECTS (European Credit Transfer System) credit.

The course presents the main concepts and mathematical apparatus of MU estimation in chemical analysis and introduces the two principal approaches to MU estimation: the ISO GUM modelling approach (the "bottom-up" or component by component approach) [8] and the single-lab validation (the "top-down") approach [9].

In spite of being introductory, the course intends to offer sufficient knowledge and skills for carrying out MU estimation for most of the common analyses in routine laboratory environments. The techniques for which there are examples or exercises include acid-base titration, Kjeldahl nitrogen determination, UV-Vis spectrophotometry, atomic absorption spectroscopy, liquid chromatography mass spectrometry (LC-MS), etc. It is important to stress that for successful measurement uncertainty estimation experience (both in analytical chemistry as such and also in uncertainty estimation) is crucial and this can be acquired only through practice.

The course materials

The course materials at https://sisu.ut.ee/measurement/ uncertainty/ are freely available to everyone all the time. The course materials consist of short video lectures (with 
slides available for downloading), practical examples and numerous tests and exercises for self-testing. The systematic presentation of measurement uncertainty in chemistry is divided into 12 chapters (occasionally divided into subchapters). A chapter has a short video lecture (sometimes several), accompanying textual material (including schemes, formulas, etc.) and in most cases one or several self-tests. The self-tests range from simple "ticking" tests to complex full-fledged MU estimation problems. The self-tests do not need registration to the course, they do not influence the final score of the registered participants and there is no limit to how many times a learner can attempt the self-tests.

The course starts with an explanation of the concept of MU (Chapter 1). Chapter 2 explains the concept of uncertainty source and explores the sources of uncertainty in the simple example of pipetting (classical volumetric pipettes as well as automatic pipettes are addressed). This chapter also introduces the concept of random and systematic effects. Chapter 3 introduces the main mathematical tools for MU: the normal distribution, mean, standard deviation, standard uncertainty, A and B type uncertainty estimates, standard deviation of the mean, rectangular, triangular and the Student distribution. Chapter 4 presents the first MU estimation (on the example of pipetting) based on identification and quantification of the individual uncertainty sources (the component by component approach).

Thus, Chapters 1-4 give the very basics of the topic, but are nevertheless sufficient for carrying out the first measurement uncertainty evaluation for a very simple case (the example of pipetting). It was considered important to present the material in such a way as to enable participants to make a simple MU evaluation as early in the course as possible.

Chapters 5-7 revisit the fundamentals of measurement uncertainty but this time with more in-depth coverage. Chapter 5 explains the main principles of MU estimation, known as the GUM principles [8]. Chapter 6 revisits the random and systematic effects on measurement result and explains that these concepts are not absolute, but depend on the selected time frame (i.e. effects that are systematic in the short term can become random in the long term). Chapter 7 gives a complete and comprehensive picture of the connections between the concepts random and systematic error, trueness, precision, accuracy, bias, repeatability, intermediate precision (withinlab reproducibility) and MU. By the end of Chap. 7 the student has all the theoretical knowledge needed for MU estimation.

Chapter 8 gives an overview of the two most widespread approaches to MU estimation: the ISO GUM modelling approach [8] and the single-lab validation approach as implemented by Nordtest [9]. Chapters 9 and 10 are the lengthiest and are devoted to these two approaches, respectively. Both approaches are presented using practical examples, which are discussed in detail. The learners can download slides and calculation files. The self-tests of these two chapters include serious MU calculation problems.

Chapter 11 gives a brief comparison of the MU estimation approaches. Chapter 12 explains how to compare two measurement results if MU estimates are available for both. Chapter 13 presents some additional materials and studies for more interested students.

\section{Course organization}

In the Moodle learning management system (the link with guest user access is available elsewhere [7]), the course chapters were divided across 6 weeks (week 1, Chaps. 1-2; week 2, Chap. 3; week 3, Chap. 4; week 4, Chaps. 5-7; week 5, Chaps. 8-9; week 6, Chaps. 10-12). During each week the students were asked to work with the respective chapters in the study materials and assess their knowledge with the selftests. Each week ended with a graded test, often composed of several parts and ranging, as the self-tests, from simple "ticking" tests to complex full-fledged MU estimation problems.

The schedule described above was suggested for the participants but was not mandatory. All the materials and graded tests were open throughout the course and more advanced participants were welcome to pass the course faster (the most advanced students required only a few days to go through all materials and all graded tests), while novices were able to use more time in the first weeks. Nevertheless, by the end of the sixth week the learners had to complete all six graded tests. Every graded test could be taken several times, ranging from three in the first weeks to ten in weeks 5 and 6 (the latter weeks had the most difficult tests, i.e. full-fledged measurement uncertainty evaluation problems). The highest obtained score counts for the final grade. For assigning the final grade the weighted sum of the scores of the different weeks is calculated and grades are assigned as follows: A, 91-100; B, 81-90; C, 71-80; D, 61-70; E, 51-60; F, less than 51.

During the 6-week course period the students were able to communicate among themselves and with teachers via forums. The course has two general forums-for general questions and technical problems - and in addition six forums for discussing the topics of the specific weeks.

In order to successfully completing the course a student was required to achieve a score of more than $50 \%$ from each graded test. In the tests with complex exercises (weeks 5 and 6) scores were assigned in large increments, e.g. two parts scoring 70 and 30 , respectively. This means that for such a test it is not possible to get a positive score of less than 70 . For this reason the overall scores obtained by participants were mostly beyond $80 \%$. The students who successfully passed the 
course were awarded a certificate from the University of Tartu (sent to them by mail).

\section{Results}

At the time of writing the course has been running once during spring 2014. The course content was ready by the end of 2013 . The registration for spring 2014 was launched on 14 January and the course began on 3 March 2014. The course eventually had 270 registered participants from more than 40 countries of whom 211 actually started learning (i.e. logged on to the learning management system at least once).

The majority of the participants were analytical chemistry practitioners. The participants were very active throughout the course and asked lots of questions via the forums. These were often very much to the point and addressed things that are really important to analysts in their everyday work. The overall number of posts to them during the course period reached beyond 300 (overall number of posts, both from participants and teachers).

This active participation made teaching this $\mathrm{MOOC}$ a great experience also for the teachers. The discussion threads gave a lot of added value to the course and some of them triggered modifications to the course materials.

The course finished on 16 April 2014 with 141 participants successfully completing the course, making the completion rate $52 \%$ with respect to all registered participants (or $67 \%$ with respect to the participants who actually started learning). We consider this completion rate very good for a MOOC, especially one that has quite difficult calculation exercises, which need to be done correctly to complete the course.

The feedback of the participants was very positive (some examples can be seen on the course website [7]) and we are glad that evidently we were able to offer something that is really needed by people who do chemical analysis in their everyday work, such as many of our participants.

\section{Discussion}

MOOCs and university courses

It is common to compare MOOCs with conventional university courses. We find that it is also useful to include the practitioner training courses in the comparison. These are typically short courses (1-3 days) for professionals, usually quite practically oriented. We have experience in teaching measurement uncertainty in analytical chemistry in all three formats. The comparison is summarized in Table 1.
Table 1 reveals that in the big picture (i.e. in the context of a full university undergraduate programme, as opposed to single courses), MOOCs are no real competitors for the conventional university education, especially not in experimental sciences, such as chemistry. Occasionally a university programme could be completed on the basis of MOOCs, e.g. in humanities. However, then the final assessment has to be carried out face-to-face: there is no way of knowing who actually completes the online tests in MOOCs. In single (usually with a low number of credits) courses such risk can be tolerated, but not in the case of awarding a university degree. This would involve some travelling but would still be cost-effective compared to the conventional university education and would enable full-time work while studying.

\section{MOOCs and practitioner training courses}

It is more interesting to compare MOOCs with short practitioner training courses. It can be seen that almost full complementarity exists between the two: the weaknesses of one are the strengths of the other.

From the learning point of view the main drawbacks of MOOCs are the limited participant-teacher and participantparticipant interaction possibilities and high selfdiscipline needed. At the same time, lack of time constraints enables deep coverage and analysis of the subject, extensive problem-solving, etc. This is especially beneficial in the case of subjects that are conceptually and mathematically nontrivial and where an important part of competence is the ability to carry out complex calculations. Measurement uncertainty estimation as a topic is an excellent example.

A situation often (in fact, usually) encountered in training courses is that the level of previous knowledge of participants is uneven. Thus, in order to enable the participation of everybody the basics also need to be explained with care. This means that some participants are bored while others struggle to stay on track. The outcome often is that the most interesting part of the training-problem-solving - receives a disproportionally small amount of time at the end of the course. We have tried to announce such training courses for "advanced" participants only, but the outcome has been only slightly better. An additional problem is the different skills of participants in using computers and software (usually spreadsheets, such as MS Excel). It is difficult to carry out serious uncertainty estimation without computers. We have asked participants to bring their own PCs, but this is only partly of help, if some participants are not fluent with spreadsheets. Helping users to work with computers and software takes time and while struggling with the PC the participants lag behind and time is very 
Table 1 Comparison of MOOCs to conventional university courses and short training courses for practitioners

\begin{tabular}{|c|c|c|c|}
\hline Aspect & Conventional university course & Practitioner training (short) course & MOOC \\
\hline Interaction between students and teachers & Direct & Direct & Remote \\
\hline $\begin{array}{l}\text { Possibility to deliver the course simultaneously } \\
\text { to many participants }\end{array}$ & Low & Low & High \\
\hline Level of self-discipline needed from participants & Average & Average & High \\
\hline Time constraints, time to "digest" the knowledge & Not a problem & Serious time constraints & Not a problem \\
\hline Possibility of independent homework & Possible & Usually impossible & Possible \\
\hline Possibility of hands-on problem-solving & Possible & Possible (within the time constraints) & Possible \\
\hline Possibility of teamwork & Possible & Possible (within the time constraints) & Not easy \\
\hline Possibility of experimental work & Easy & Possible, but not easy & Not possible \\
\hline $\begin{array}{l}\text { Possibility of working with participants of uneven } \\
\text { level or preparation }\end{array}$ & Difficult but possible & Difficult & Possible \\
\hline $\begin{array}{l}\text { Possibilities of meaningful assessment of } \\
\text { obtained knowledge }\end{array}$ & Wide possibilities & Difficult & Possible \\
\hline Danger of cheating during knowledge assessment & Can be made low & Can be made low & Can be high \\
\hline Costs of setting up the course ${ }^{a}$ & Medium & Medium & Medium \\
\hline Costs of running the course $\mathrm{e}^{\mathrm{a}}$ & High & High & Low \\
\hline Travel and accommodation costs & Can be high & Can be high & None \\
\hline
\end{tabular}

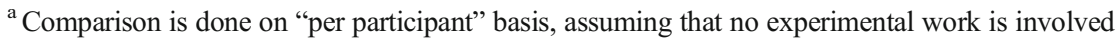

limited. These problems are dramatically reduced under MOOC conditions when every participant can work at their own pace. In our uncertainty MOOC the coverage of uncertainty starts from the very basics and the first weeks are less intense than the last, so that people with very little background can successfully participate. At the same time, more advanced users can work through the first weeks fast and concentrate on the more challenging topics of the subsequent weeks. This possibility for everyone to work at their own pace has been specifically outlined in several participant feedbacks as a strong point.

When it comes to evaluation of obtained knowledge then in the typical practitioner training course-lasting for two full days (very common for short practitioner training courses) with a knowledge assessment test at the end of the second day-meaningful assessment of acquired knowledge is not possible. The participants have not had the possibility to digest the obtained knowledge; however, in the case of MOOCs, digestion is possible, as well as asking guidance during preparation for the tests. In the case of our MOOC the knowledge assessment is spread over the whole course period and the number of attempts allowed is quite large. Therefore there is no nervousness or the necessity to take a single test covering a very large amount of material.

Of course, MOOC-based teaching is possible with very large groups of learners at low running cost (per participant) and at zero travel/accommodation cost.
Different possibilities of using the online materials

The MOOC described here was created first of all for sharing the competence in metrology in chemistry that has been acquired at UT Department of Analytical Chemistry to practitioners and to make UT a better known study destination for student candidates from the whole world. During working with the MOOC additional ideas emerged as how to make use of the teaching materials that have been created. Figure 1 presents the usage possibilities. It can be seen that the investment in the online teaching materials brings back much more benefit than using the materials just for MOOCs.

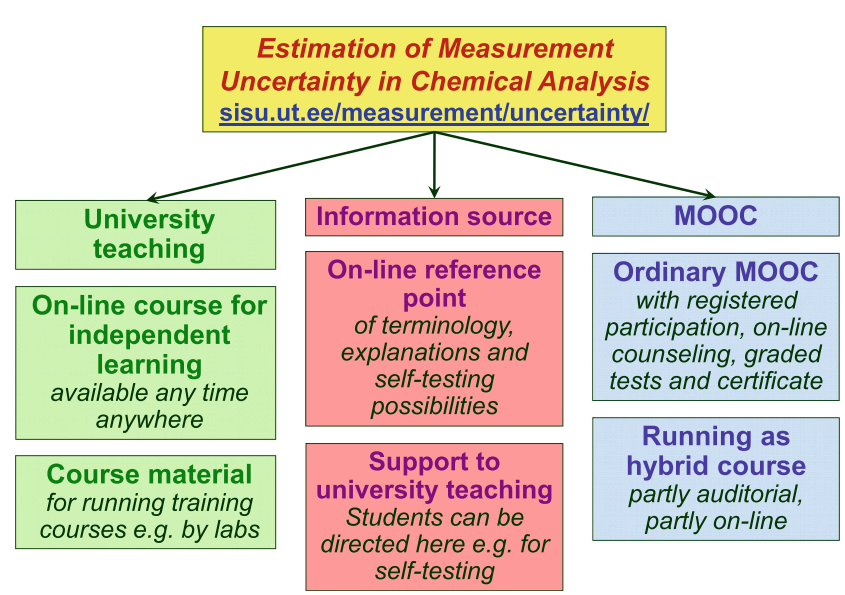

Fig. 1 Modes of usage of the course materials of the MOOC "Estimation of measurement uncertainty in chemical analysis" 


\section{Conclusions}

Our experience with the MOOC on measurement uncertainty in analytical chemistry suggests that in the field of measurement uncertainty in chemistry (or in chemistry education in general) MOOCs are no real competitors for the conventional university degree programs. Instead they can rather be seen as useful add-ons. At the same time in the context of practitioner training, online courses can offer significant advantages over the conventional intensive course format lasting 1-3 days. The online teaching materials of a MOOC can find many uses, e.g. as supporting materials for conventional university teaching.

Acknowledgments The authors are thankful to Triin Marandi, Enno Kaasik, Lehti Pilt and Esta Pilt for their kind and professional technical support in setting up and running this course.

\section{References}

1. Cormier D (2008) The CCK08 MOOC - connectivism course, $1 / 4$ way. Available from Dave Cormier's blog. http://davecormier.com/ edblog/2008/10/02/the-cck08-mooc-connectivism-course-14-way/. Accessed 2 Jan 2015

2. Pappano L (2012) The year of the MOOC. The New York Times Nov 2, 2012

3. Lewin T (2013) Setbacks force new look at mass web courses. International New York Times Dec 12, 2013

4. Skapinker M (2013) Open web courses are massively overhyped. Financial Times Mar 13, 2013

5. Coursera Inc. (2014) https://www.coursera.org/. Accessed 2 Jan 2015

6. edX Inc. (2014) https://www.edx.org/. Accessed 2 Jan 2015

7. University of Tartu (2013) Estimation of measurement uncertainty in chemical analysis, online course. https://sisu.ut.ee/measurement/uncertainty/. Accessed 2 Jan 2015

8. JCGM 100:2008 Evaluation of measurement data - guide to the expression of uncertainty in measurement (GUM 1995 with minor corrections). Available from http://www.bipm.org/. Accessed 2 Jan 2015

9. Magnusson B, Näykki T, Hovind H, Krysell M (2012) Handbook for calculation of measurement uncertainty in environmental laboratories. Nordtest technical report 537, ed. 3.1 Nordtest. Available from http://www.nordtest.info/. Accessed 2 Jan 2015

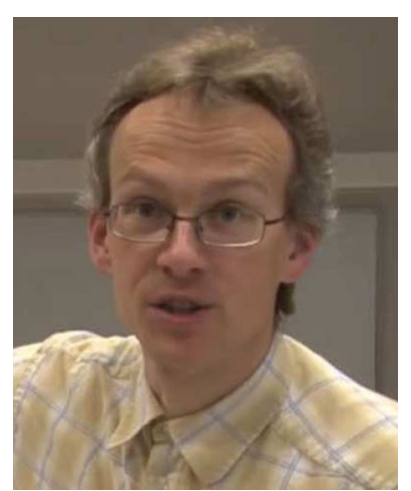

Ivo Leito works as professor of analytical chemistry at University of Tartu. His research directions are on the borderline of analytical chemistry with other disciplines: chemistry of superacids and superbases; metrology in chemistry (MiC); liquid chromatography and mass spectrometry; sensors and their metrological characterization; applications of instrumental methods in analysis of historical objects. He teaches analytical chemistry and its metrological aspects at UT and has been involved in setting up several international MiC-related educational activities.

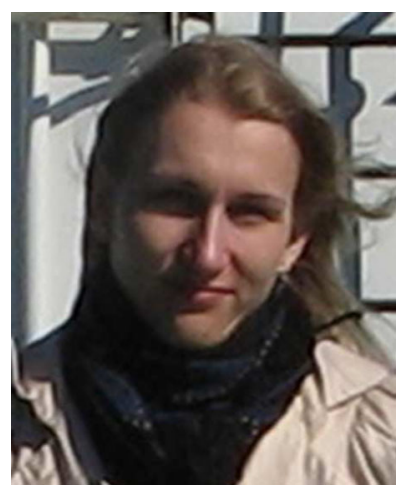

Irja Helm is an analytical chemistry research fellow at University of Tartu. She teaches practical classes of analytical chemistry. She takes care that metrological concepts and approaches are introduced to students at as early stage of analytical chemistry studies as possible. Her research is focused on high-accuracy reference measurements of dissolved oxygen concentration.

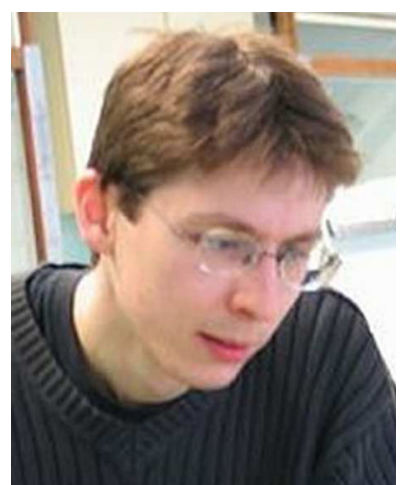

Lauri Jalukse is analytical chemistry research fellow at University of Tartu. He teaches analytical chemistry and metrology in chemistry at all study levels. He is continuously introducing innovative and active learning approaches into teaching. His research work is focused on metrological studies of electrochemical and optical sensors, measurements of dissolved oxygen concentration and moisture content, as well as organization of interlaboratory comparisons. 